\title{
Phasic alerting facilitates endogenous orienting of spatial attention: Evidence from event-related lateralizations of the EEG
}

\author{
Dariusz Asanowicz $^{1}$ (D) Bartłomiej Panek ${ }^{1}$
}

Published online: 6 January 2020

(C) The Psychonomic Society, Inc. 2020

\begin{abstract}
Alerting has been hypothesized to affect spatial orienting either by accelerating the speed of attentional shift toward the cued target location (the accelerating hypothesis) or by enhancing the orienting effect without changing its time course (the enhancing hypothesis). To investigate the neural underpinnings of the effect of phasic alerting on endogenous orienting, we recorded the electroencephalogram (EEG) in a variant of the spatial cueing task with a tone presented $100 \mathrm{~ms}$ before the cue as a phasic alerting signal, and calculated cue-evoked event-related lateralizations (ERLs) providing a precise assessment of preparatory visuospatial attention. Behavioral results showed that the spatial orienting effect was increased under the phasic alerting condition, as expected. The EEG results showed that an orienting-related ERL component called a late directing attention positivity (LDAP) had shorter onset latency and larger amplitude in the alerting condition than in the no-alerting (no-tone) condition. In conclusion, phasic alerting seems to both accelerate and enhance orienting-related preparatory modulations within the ventral visual stream.
\end{abstract}

Keywords Phasic alerting $\cdot$ Spatial orienting $\cdot$ Event-related lateralization $\cdot$ ERL $\cdot$ Late directing attention positivity $\cdot$ LDAP

According to Posner and colleagues, alerting and orienting are two relatively independent components of the human attention system (Petersen \& Posner, 2012; Posner \& Rothbart, 2007). Alerting has been defined as a mechanism of achieving a state of readiness to process and respond to external stimuli (Posner, 2008), and orienting as a mechanism of shifting and focusing attention on spatial locations or objects of interest to select relevant information (Posner, 2014). It is well known that both alerting and orienting increase the speed of responding to target stimuli. Response time (RT) is faster when a target is preceded by a warning stimulus (e.g., a short accessory tone), compared with RT to a target occurring without any warning, which is interpreted as an effect of phasic alertness increasing perceptual sensitivity and thus reducing stimulus-encoding time (alerting effect; Hackley \& ValleInclán, 2003; Nobre, Rohenkohl, \& Stokes, 2012). Similarly, RT is faster when a target is preceded by a cue providing information about the target location, compared with RT to a

Dariusz Asanowicz

d.asanowicz@uj.edu.pl

1 Institute of Psychology, Jagiellonian University, Ingardena 6, 30-060 Krakow, Poland target occurring either at an uncued location or preceded by a spatially neutral cue, which is interpreted as a result of more efficient processing of attended than unattended stimuli (orienting effect; Carrasco, 2011; Corbetta \& Shulman, 2002).

The results of a study by Fernandez-Duque and Posner (1997) showed that the effects of alerting and orienting on target processing may occur additively - that is, with no interactions - in agreement with the presumed functional and neuronal independence of the two mechanisms. Notwithstanding, the notion of independence does not imply that there are no interactions between them. Alerting practically by definition is assumed to affect the orienting process (Posner \& Fan, 2008), and indeed, alerting and orienting have been shown to interact. When the alerting tone is presented shortly before the orienting cue, the RT cueing effect increases, indicating that phasic alerting facilitates the orienting of attention toward the target location (Callejas, Lupiàñez, Funes, \& Tudela, 2005; Callejas, Lupiáñez, \& Tudela, 2004; Fuentes \& Campoy, 2008; Ishigami et al., 2016). It has also been shown that phasic alerting improves deficits in orienting to left-sided targets in patients with hemispatial neglect (Chica et al., 2012). Yet the exact mechanism of this interaction is still not well understood (Posner, 2012).

Two hypotheses have been put forward (Callejas et al., 2005; Posner, 1978). Alerting may speed up the orienting 
effect without increasing its magnitude so that the maximum of the orienting effect would be reached earlier than in the noalerting condition, but this maximum would be the same regardless the level of alertness (the accelerating hypothesis). Alternatively, alerting may increase the magnitude of the orienting effect, but without affecting its time course, so that the effect maxima would be reached with the same latency regardless the alerting conditions, but the benefit gained from the spatial cueing would be larger under the alerting condition (the enhancing hypothesis). The results obtained by Callejas et al. (2005) have supported the accelerating hypothesis. They found that alerting increased the orienting effect when the interval between cue and target was short (100 ms), but the interaction disappeared when the interval was longer (500 $\mathrm{ms}$ ), presumably because "when more time is allowed (long SOA), the system is able to effectively orient to the target independently of the presence or absence of an alerting signal" (Callejas et al., 2005, p. 34). However, a subsequent study by Fuentes and Campoy (2008) has shown that the alertingorienting interaction was mostly independent of the cuetarget interval, which contradicts the accelerating hypothesis and supports the enhancing hypothesis.

The aim of the present study was to determine the neural underpinnings of the effect of phasic alerting on endogenous orienting. We used a spatial cueing task in which a target, presented in the left or right visual field, was preceded by a central cue, which was either an arrow indicating the target location (valid cue) or a square providing no information about the target location (neutral cue). Alerting was induced by presenting an accessory tone before the cue, with a 100 or 500-ms tone-cue onset asynchrony (TCOA) interval. As phasic alerting is assumed to be a fast, exogenous, and shortlasting process, its effect on orienting was expected to be captured under the condition with the short TCOA (Asanowicz \& Marzecová, 2017; Weinbach \& Henik, 2013). The longer TCOA was introduced to diminish temporal predictability of the tone, thereby to prevent confounding phasic alerting with temporal orienting (Weinbach \& Henik, 2012a). To investigate the time course of the effect of phasic alerting on spatial orienting, we recorded the electroencephalogram (EEG) and computed so-called event-related lateralizations (van der Lubbe, Neggers, Verleger, \& Kenemans, 2006; Wascher \& Wauschkuhn, 1996). The ERL is a contralateral minus ipsilateral difference derived from event-related potentials (ERPs) recorded at the hemispheres contralateral and ipsilateral to the cued visual fields. The crucial feature of the ERL is that all bilateral and central activity (including activity related to perceptual processing of the tone and centrally presented cue), and general hemispheric differences are subtracted out (Gratton, Coles, Sirevaag, Eriksen, \& Donchin, 1988; Wascher \& Wauschkuhn, 1996), thereby providing a precise assessment of spatially specific changes evoked in response to the attention-directing cues. Several preparatory orienting-related ERL components have been distinguished thus far, including an early directing attention negativity (EDAN) that presumably reflects the first stages of cuedirected orienting, and a late directing attention positivity (LDAP) reflecting orienting-related preparatory modulations within the ventral visual stream (Harter et al., 1989; Hopf \& Mangun, 2000; van der Lubbe et al., 2006; Wascher \& Wauschkuhn, 1996).

If the accelerating hypothesis holds true, phasic alerting should decrease latencies of the orienting-related ERL components without changing their amplitudes. Alternatively, we may observe larger ERL amplitudes under the phasic alerting condition with no changes in the time course of the components, in line with the enhancing hypothesis.

\section{Method}

\section{Participants}

Fifty-five undergraduate students took part in the experiment in return for course credits. All participants had a normal or corrected-to-normal vision and no history of neurological disorders. Informed written consent was obtained before the experiment. Data from three participants were excluded from analysis due to error rates (ERR) exceeding two standard deviations of the whole group, data from two participants were excluded because of large EEG artifacts, and data from seven participants were excluded because of horizontal eye movements (see below for details). Mean age of the final sample ( $N$ $=43,35$ females) was 20.2 years $(S D=2.4)$.

\section{Stimuli, apparatus, and procedure}

The task is illustrated in Fig. 1. Each trial of the task began with a fixation point displayed at the center of the computer screen. The time of the initial fixation varied randomly between 1,000 and 2,000 ms (in $150 \mathrm{~ms}$ steps, distributed uniformly). The fixation point was continuously displayed during the whole trial (except when it was replaced by the cues). The target stimulus was an arrow pointing either up or down, presented in the left or right visual field (50/50). For reasons not of interest to the present research question, the target arrow was flanked by four additional arrows pointing in either the same or the opposite direction (i.e., congruent and incongruent flankers, 50/50), and a corresponding distractor set consisted of five vertical lines without arrowheads was simultaneously presented in the visual field opposite to the target. These stimuli were presented for $180 \mathrm{~ms}$. In each trial, the target stimulus was preceded by a valid or neutral visual cue $(50 / 50)$, presented at the center of the screen for $100 \mathrm{~ms}$, with $600 \mathrm{~ms}$ cuetarget onset asynchrony (cf. Harter et al., 1989). The valid cue was a double arrow sign pointing left or right: " $<<$ " or " $>>$ " 


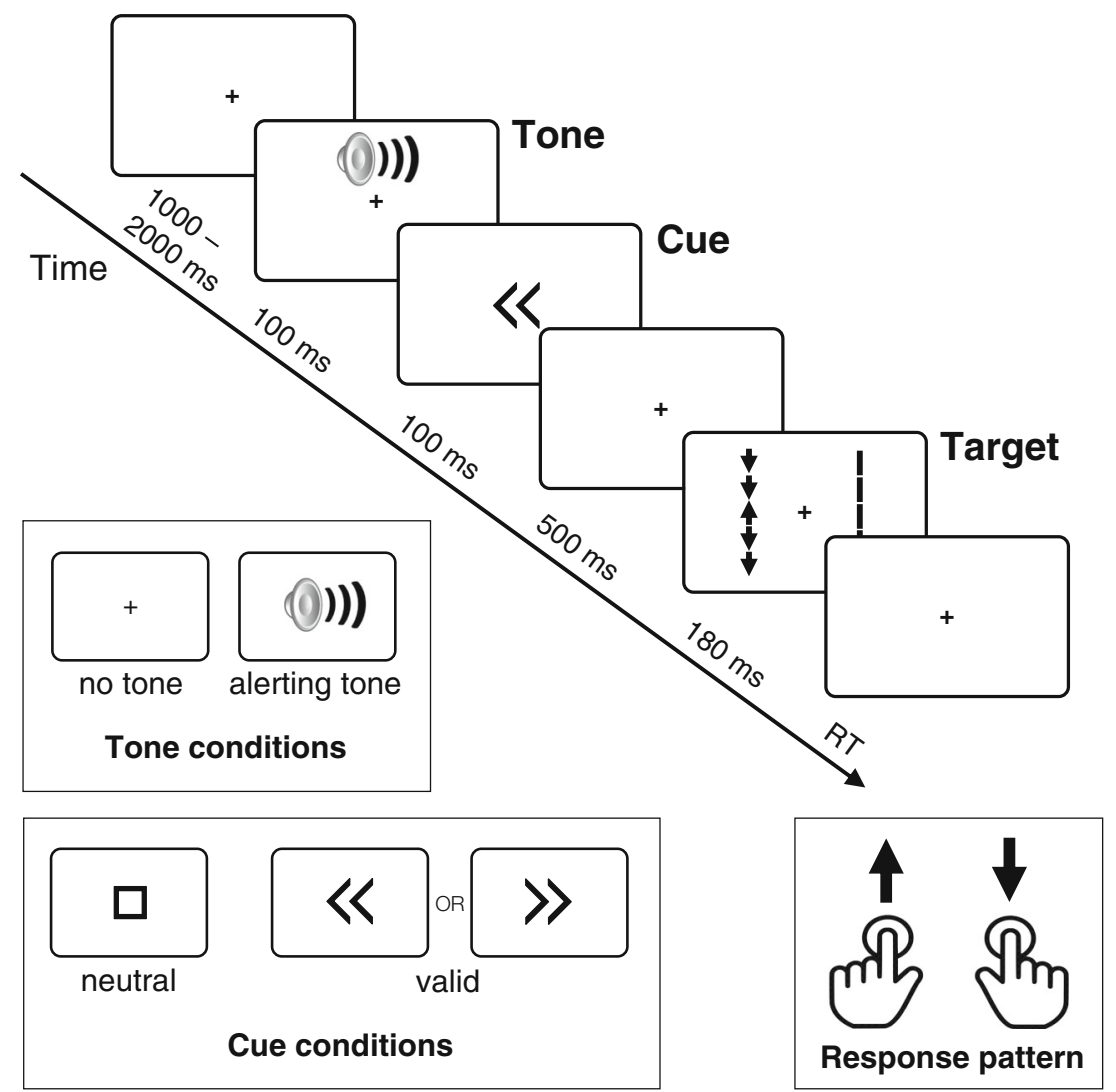

Fig. 1 The stimuli used in the task and sequence of events in a trial. The example shows an alerting tone $(2000 \mathrm{~Hz}, 50 \mathrm{~ms})$ presented $100 \mathrm{~ms}$ before onset of a valid spatial cue indicating the spatial location of an upcoming target. See Method section for details

(50/50), whereas the neutral cue was a square composed of the four arms of the two arrows. Thereby, in terms of the physical properties of stimuli, the neutral and valid cues were exactly the same. The valid cue always correctly indicated the target location. In two thirds of all trials, a $2000 \mathrm{~Hz}, 50$-ms alerting tone was presented before the cue, with 100 or 500 -ms tonecue onset asynchrony (TCOA). All these variables were counterbalanced. Condition order was randomized per participant.

The fixation cross was $4 \mathrm{~mm}\left(0.4^{\circ}\right)$ wide. The length of the side of the square (neutral cue) and of the one arm of the cue arrow (valid cue) were $4 \mathrm{~mm}\left(0.4^{\circ}\right)$. The height and width of the two arrows together (valid cue) were $5 \mathrm{~mm}\left(0.5^{\circ}\right)$. The target arrow and the flankers were each $8-\mathrm{mm}\left(0.8^{\circ}\right)$ long. The target and flankers' arrowheads were 4 -mm wide $\left(0.4^{\circ}\right)$. The length of all five (target and flanker) arrows in the display was $44 \mathrm{~mm}\left(4.4^{\circ}\right)$. The arrows' midpoints were displayed $18.5 \mathrm{~mm}$ $\left(1.85^{\circ}\right)$ to the left or right of the center of the screen. All stimuli were black and were presented on a light-gray background (RGB: 245, 245, 245). PsychoPy software (www. psychopy.org) was used for experimental control.

The participants' task was to identify the direction of the target (middle) arrow and respond by pressing the left or right Ctrl key on the computer keyboard with the left or right index finger, respectively, to the up-pointing and down-pointing target arrows. Speed and accuracy of responses were measured. A new trial began automatically after the response or after 2,000 ms if the participant did not respond. Participants were given written and then verbal instructions describing the task. They were asked to respond to target stimuli as quickly and accurately as possible, and to direct their attention to the location indicated by the arrow cues to respond even faster and more accurately. They were also carefully instructed to keep central fixation and explained why proper fixation is necessary during EEG measurement. The task began with two practice blocks consisting of 32 trials in total in which participants received accuracy feedback after each response. The practice session was followed by 576 experimental trials with no feedback divided into four blocks of 144 trials. The order of the trials was randomized within blocks individually for each participant. Between the blocks, participants were asked to take breaks to rest their eyes. The task lasted up to 1 hour. The whole session lasted up to 90 minutes.

\section{Response-time analysis}

Trials with an incorrect response, or with RT below $200 \mathrm{~ms}$ or above 1,200 ms, were excluded from the analyzes (overall $4.8 \%$ ). Correct response times were first submitted into an omnibus $3 \times 2$ repeated-measure ANOVA with tone (no- 
tone, tone with TCOA $100 \mathrm{~ms}$, tone with TCOA $500 \mathrm{~ms}$ ) and cue (valid, neutral) as within-subjects factors, and then the tone effects in the two TCOA conditions were examined separately by $2 \times 2$ repeated-measure ANOVAs. The Greenhouse-Geisser correction was applied when the tone factor had three levels. The effects of phasic alerting were assumed to be captured only with the TCOA $100 \mathrm{~ms}$ (Asanowicz \& Marzecová, 2017; Weinbach \& Henik, 2013), and thus the alerting $\times$ orienting interaction was expected to be nonsignificant with the TCOA $500 \mathrm{~ms}$. This was confirmed (see RT Results), thus further analyzes were focused on the no-tone and TCOA $100 \mathrm{~ms}$ conditions, aiming specifically at the effects of phasic alerting.

\section{EEG data recording and preprocessing}

EEG was recorded using a BioSemi ActiveTwo system with $\mathrm{Ag}-\mathrm{AgCl}$ electrodes on 64 monopolar locations according to the extended 10-20 system, and two additional electrodes, the common mode sense (CMS) active electrode and the driven right leg (DRL) passive electrode, used as reference and ground electrodes, respectively (www.biosemi.com/faq/ cms\&drl.htm). All cephalic electrodes were placed on the scalp using the Electro-Cap. Vertical electrooculogram (EOG) was recorded from above and below the left eye, and horizontal EOG was recorded from the external canthi of both eyes. The data was stored at a sampling rate of $512 \mathrm{~Hz}$.

Brain-Vision Analyzer software (Version 2, Munich, Germany) was used for off-line data processing. Data were filtered with a $0.1-50 \mathrm{~Hz}$ band-pass and a $50 \mathrm{~Hz}$ bandrejection filter (Butterworth zero-phase filters, attenuation of $12 \mathrm{~dB}$ /octave), rereferenced to linked mastoids, and split into segments from $200 \mathrm{~ms}$ before tone onset to $1,000 \mathrm{~ms}$ after cue onset. Segmented data were referred to the first $200 \mathrm{~ms}$ of the segment as baseline. Trials with an incorrect response, or with RTs shorter than $200 \mathrm{~ms}$ or longer than 1,200 ms were automatically excluded.

Data with horizontal eye movements were excluded in two steps. First, segments were marked if horizontal EOG activity (right minus left EOG) exceeded $\pm 35 \mu \mathrm{V}$ and/or voltage steps between adjacent data points exceeded $5 \mu \mathrm{V}$, and then removed after visual inspection if a distinctive "boxcar" shape, characteristic for saccadic eye movements, was found in these marked segments within the cue-target interval. Participants for whom more than $30 \%$ of trials were rejected at this step were excluded from further analysis. In the remaining sample, on average, $6 \%$ of segments (range: $1 \%-28 \%$ ) were removed because of horizontal eye movements. Next, we calculated averaged horizontal EOG waveforms contralateral minus ipsilateral to the left-pointing and right-pointing cues in the valid cue trials to assess whether any residual hEOG activity was present in the remaining data. Participants for whom these averaged hEOG difference waves exceeded $6.4 \mu \mathrm{V}$ within cue-target interval, indicating eye movements $\geq 0.2^{\circ}$ toward the cued location, were excluded from further analysis. In sum, data of seven participants were excluded because of horizontal eye movements. The grand average of the hEOG activity (right minus left EOG) in the final sample is shown in the upper panel of Fig. 3.

Before ocular correction, gross artifacts at the scalp electrodes were first removed by rejecting segments with overall minimum-maximum voltage differences $\geq 200 \mu \mathrm{V}$ or with voltage steps between adjacent data points $\geq 80 \mu \mathrm{V}$ (except AF3, AFz, AF4, Fp1, Fpz, and Fp2, so that fewer segments would be rejected due to blinks). Next, the data from remaining trials were corrected for eye blinks and artifacts that had a noncortical origin using independent component analysis (ICA; Jung et al., 2000), and then, after resetting the baseline, edited for other artifacts by rejecting trials with overall minimum-maximum voltage differences $\geq 120 \mu \mathrm{V}$, voltage steps between adjacent data points $\geq 20 \mu \mathrm{V}$, and absolute amplitudes $\geq 120 \mu \mathrm{V}$. On average $7 \%$ of segments (range: $1 \%$ $14 \%$ ) were excluded because of artifacts.

\section{Event-related lateralization (ERL) analysis}

Artifact-free and eye-movement-free segments were averaged per participant over the relevant conditions separately for the left and right targets. Next, ERLs were calculated for all symmetrical electrodes as the average of contralateral minus ipsilateral differences for the left-pointing and right-pointing cues in the valid cue condition, and left and right targets for the neutral cue condition, by the formula: $\left(\left(\mathrm{RH}_{\mathrm{LVF}}-\mathrm{LH}_{\mathrm{LVF}}\right)+\left(\mathrm{LH}_{\mathrm{RVF}}-\mathrm{RH}_{\mathrm{RVF}}\right)\right) / 2$ (van der Lubbe et al., 2006; Wascher \& Wauschkuhn, 1996). Thus, in the valid cue condition, a positive value indicates larger positivity and a negative value indicates larger negativity at the hemisphere contralateral to the cued location, reflecting cue-direction-related activity. In the neutral cue condition, the ERLs should amount to zero.

Based on previous ERL studies and on inspection of the time course and topographies of the obtained ERLs (shown in Fig. 3), we identified five ERL components in the valid cue conditions. The first three were classified as cue-processing-related N1pc, P1pc, and N2pc components, and another two were classified as the expected orienting-related EDAN and LDAP components. The cue-processing-related ERLs have been considered not relevant for the present research question (see Discussion), thus, only the EDAN and LDAP analyses are reported, for brevity. Average EDAN and LDAP amplitudes were computed from the occipitotemporal PO7-8 and P7-8 electrode pairs, where the ERLs were most prominent (see topographies on Fig. 3; for similar results, see, e.g., Lasaponara, Chica, Lecce, Lupianez, \& Doricchi, 2011; van der Lubbe, de Kleine, \& Rataj, 2019; 
van der Lubbe et al., 2006). The amplitudes were measured per participant as mean voltage $300-340 \mathrm{~ms}$ (EDAN) and 380-600 ms (LDAP) after cue onset, and submitted into repeated-measures ANOVAs with the factors tone (tone, no-tone), electrodes (PO7-8, P7-8), and intercept (indicating whether the difference waves differed significantly from zero; cf. Talsma, Wijers, Klaver, \& Mulder, 2001; van der Lubbe et al., 2006). One-tailed $p$ values are reported for intercept, as zero is the reference value here. To improve the signal-to-noise ratio, latencies were determined using the jackknife method: the parameters were measured from PO7-8 and P7-8 electrode pairs in one-leave-out grand means, and the diminished error variance was corrected by dividing $F$ values by $(N-1)^{2}$ (Kiesel, Miller, Jolicoeur, \& Brisson, 2008; Ulrich \& Miller, 2001). The EDAN peaks were well defined, and thus their latencies were measured by automatic peak detection within 280-360 ms after cue onset. The LDAP had a more prolonged, flattened, and irregular shape. Thus, we determined the onset of the LDAP (instead of peak latency) by means of the $30 \%$ area measure - that is, by assessing the time point that divides the first $30 \%$ of positive area under the LDAP waveform (within 340-600 ms after cue onset) from the last 70\% (Kiesel et al., 2008; Luck, 2014). We used the 30\% area measure, instead of $50 \%$, because relevant here is the question when the orienting process begins, and not when it reaches its peak. The latencies were submitted into repeated-measures ANOVAs with the factors tone (notone, tone) and electrodes (PO7-8, P7-8).

\section{Results}

\section{RT results}

The overall mean RT was $642 \mathrm{~ms}(S D=86 \mathrm{~ms})$ and mean ERR was $4 \%(S D=3 \%)$. Mean RT and ERR for each condition are shown in Table 1. In line with our assumption that the effects of phasic alerting on orienting will be observed only

Table 1 Average response time (RT) of correct responses and average error rate (ERR) for each task condition

\begin{tabular}{llll}
\hline Tone conditions & Cue conditions & RT $(S D)$ & ERR $(S D)$ \\
\hline No tone & Neutral & $661 \mathrm{~ms}(86)$ & $6 \%(4)$ \\
& Valid & $637 \mathrm{~ms} \mathrm{(82)}$ & $4 \%(4)$ \\
Tone (TCOA 100 ms) & Neutral & $656 \mathrm{~ms} \mathrm{(87)}$ & $4 \%(3)$ \\
& Valid & $622 \mathrm{~ms} \mathrm{(84)}$ & $4 \%(3)$ \\
Tone (TCOA 500 ms) & Neutral & $652 \mathrm{~ms} \mathrm{(85)}$ & $3 \%(3)$ \\
& Valid & $623 \mathrm{~ms} \mathrm{(88)}$ & $3 \%(3)$ \\
\hline
\end{tabular}

with the TCOA $100 \mathrm{~ms}$, the omnibus $3 \times 2$ interaction between tone and cue was significant $F(2,84)=4.36, p=.017$, and the $2 \times 2$ interaction between tone and cue was not significant for the TCOA $500 \mathrm{~ms}, F(1,42)=1.62, p=.21$.

The $2 \times 2$ ANOVA for the no-tone and tone TCOA 100 conditions showed that responses were generally faster in the valid cue trials than in the neutral cue trials ( 629 vs. $659 \mathrm{~ms}), F(1,42)=57.47, p<.001$, and in the tone trials than in the no-tone trials (639 vs. $649 \mathrm{~ms}), F(1,42)=$ $15.44, p<.001$. Importantly, the orienting effect (valid cue vs. neutral cue) was larger in the tone trials than in the no-tone trials ( 35 vs. $24 \mathrm{~ms}$ ), $F(1,42)=9.05, p=$ .004 . These three effects are shown in Fig. 2.

\section{ERL results}

Figure 3 shows grand averages and topographies of the ERLs for the two relevant valid cue conditions: with the alerting tone presented $100 \mathrm{~ms}$ before the cue (red line), and without the tone (black line). The middle panel of Fig. 3 also depicts the grand average for the neutral cue (control) condition (gray dashed line) - that is, the difference between sites contralateral and ipsilateral to the left and right targets. No ERLs within

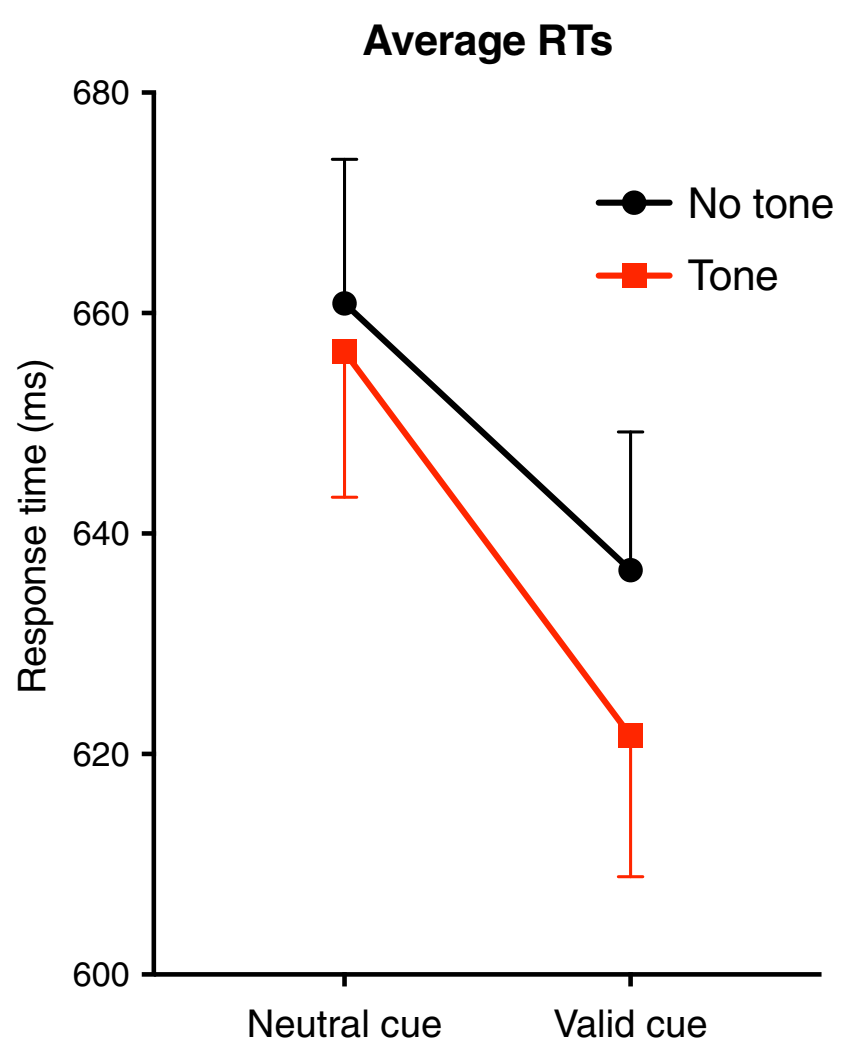

Fig. 2 Mean RTs as a function of alerting tone and orienting cue conditions. The orienting effect (neutral cue-valid cue) was increased under the phasic alerting condition (i.e., when the tone was presented $100 \mathrm{~ms}$ before cue onset), compared with the no-tone condition. Vertical bars represent standard errors of the mean 


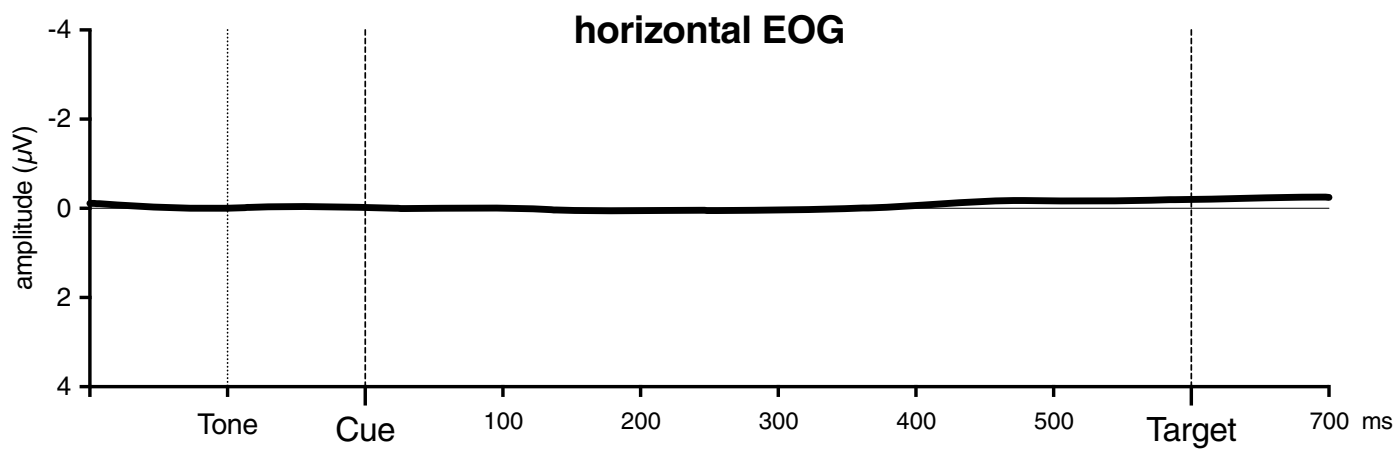

\section{Event-related lateralizations (PO7-8/P7-8)}
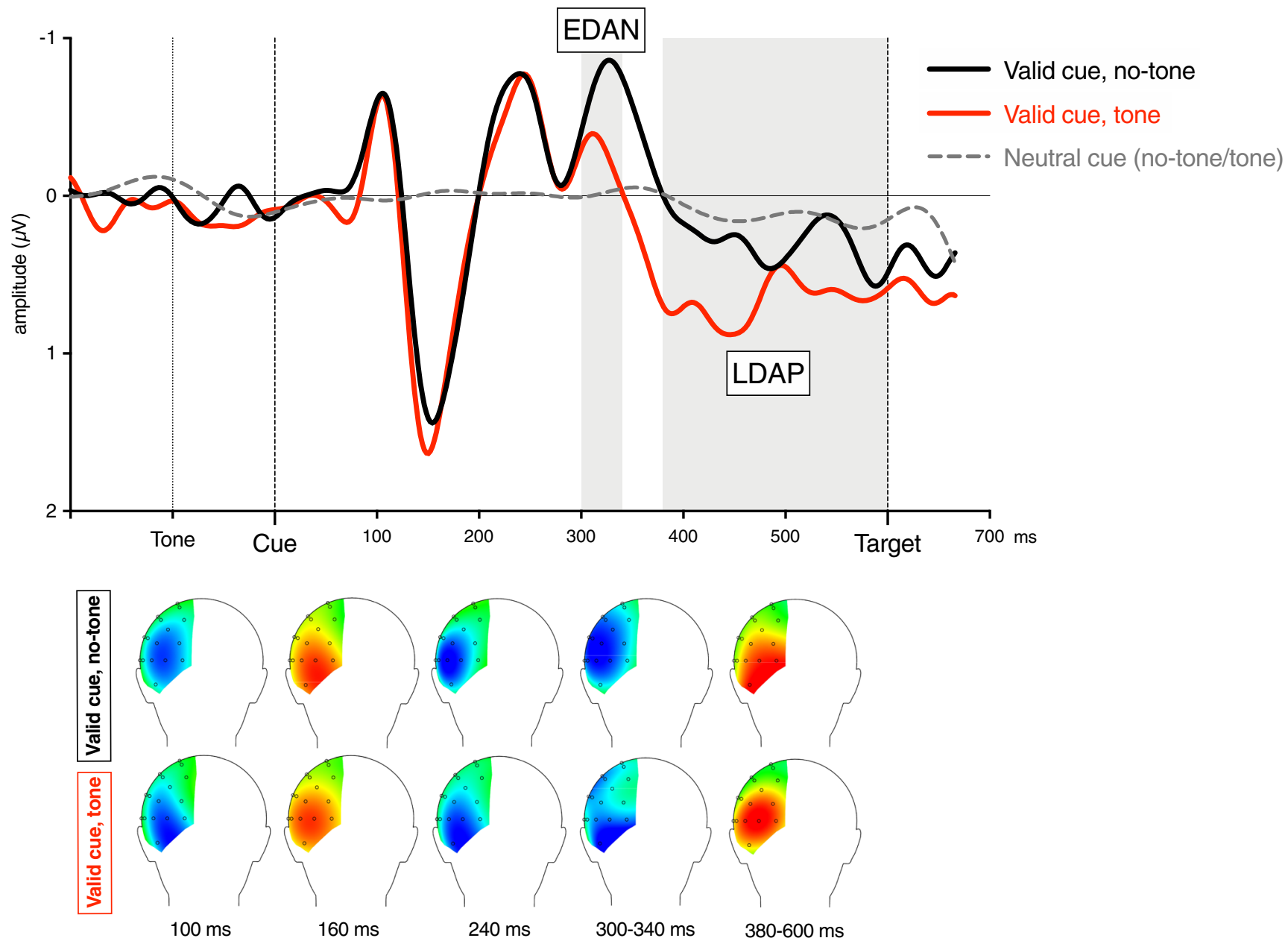

Fig. 3 Upper panel: The averaged horizontal EOG contralateral minus ipsilateral to the cued visual field, recoded so that eye movements toward both the left and right visual field would be indicated by a negative potential, and pooled across left- and right-pointing cues. The hEOG shows no systematic eye movements toward the cued locations in the final data sample. See Methods for details. Middle panel: Grand averages of the cuelocked event-related lateralizations (ERLs) for the relevant experimental conditions, pooled across two occipitotemporal electrode pairs, PO7-8 and P7-8. The ERLs were calculated as the average of contralateral minus ipsilateral differences for left-pointing and right-pointing cues in the valid cue condition, and, as a control condition, for left and right targets in the neutral cue condition. Positive values (plotted downward) and negative values indicate, respectively, larger positivity and negativity contralateral to the cued locations. The solid lines represent the cue direction-related activity under the no-alerting condition (no-tone; black line) and the phasic alerting condition (tone with TCOA $100 \mathrm{~ms}$; red line). The gray dashed line shows the ERL in the neutral cue trials (a control condition) pooled across the two tone conditions. Two ERL components related to cue-directed preparatory orienting were found: an early directing attention negativity (EDAN) and a late directing attention positivity (LDAP). The ERL in the neutral cue condition shows no cue-related spatial shifts of attention, as expected. The gray areas indicate the time windows in which the EDAN and LDAP mean amplitudes were measured (see Method and Results sections for details). Lower panel: Topographies of the consecutive ERL components. The maps are min-max scaled, with positive polarity in red, negative polarity in blue. The head view is from the back. (Color figure online) 
the cue-target interval were found in this condition, as expected. In the valid cue trials, the first three ERL components were very similar in both tone conditions forming first a negative peak at about $100 \mathrm{~ms}$, a positive peak at about $160 \mathrm{~ms}$, and again a negative peak at about $220 \mathrm{~ms}$ after cue onset, all centered at PO7-8 (tone effects: $F \leq 1.1, p \geq .31$ ). We classified these ERLs as cue processing-related N1pc, P1pc, and N2pc components, and as such considered them not relevant for the present research question (see Discussion for details). The early ERLs were followed by a negative peak at about 320 $\mathrm{ms}$, and a positive deflection that emerged at about 340-380 $\mathrm{ms}$ and outlasted target onset. The former had a broader, occipitoparietal distribution, while the latter was centered at the occipitotemporal PO7-8 and P7-8 sites. These two components fit the description (in terms of both their time course and topography) of two preparatory orienting-related ERLs: the early directing attention negativity (EDAN) and the late directing attention positivity (LDAP). Further analyses were therefore performed for the time windows from 300 to $340 \mathrm{~ms}$ and from 380 to $600 \mathrm{~ms}$ (i.e., to target onset), corresponding to the EDAN and LDAP, respectively.

EDAN

The EDAN amplitude was smaller in the tone TCOA 100 condition than in the no-tone condition, $F(1,42)=4.32, p=$ $.044, \eta_{\mathrm{p}}{ }^{2}=.09$, but remained larger than zero in the both conditions, $F(1,42)=2.85, p=.045, \eta_{\mathrm{p}}{ }^{2}=.06$, and $F(1,42)$ $=19.64, p<.001, \eta_{\mathrm{p}}{ }^{2}=.32$, respectively. The main effect of electrodes and the tone $\times$ electrodes interaction were not significant, $F \leq 1.49, p \geq .23$. Figure 3 suggests that the EDAN peaked earlier in the tone condition than in the no-tone condition, and this latency difference ( $316 \mathrm{vs} .330 \mathrm{~ms}$ ) turned out to be marginally significant, $F(1,42)=3.92, p=.051, \eta_{\mathrm{p}}{ }^{2}=.09$. The main effect of electrodes and the tone $\times$ electrodes interaction were again not significant $\left(F_{\mathrm{S}}<1.0\right)$. No significant effects of tone were found when the tone was presented with TCOA $500 \mathrm{~ms}, F \leq 1.81, p \geq .17$.

\section{LDAP}

The LDAP amplitude (unlike the EDAN) was significantly larger in the tone TCOA 100 condition than in the no-tone condition, $F(1,42)=6.52, p=.014, \eta_{\mathrm{p}}{ }^{2}=.13$, and (like the EDAN) was larger than zero both in the former, $F(1,42)=31.06, p<.001, \eta_{\mathrm{p}}{ }^{2}=.42$, and in the latter condition, $F(1,42)=5.77, p=.010, \eta_{\mathrm{p}}{ }^{2}=.12$. The main effect of electrodes and the tone $\times$ electrodes interaction were not significant $\left(F_{\mathrm{S}}<1.0\right)$. As can be seen in Fig. 3, the LDAP emerged conspicuously earlier in the tone than in the notone condition. This onset difference was estimated to be about $37 \mathrm{~ms}$ (430 vs. $467 \mathrm{~ms}$ ), and it was statistically significant, $F(1,42)=5.55, p=.023, \eta_{\mathrm{p}}{ }^{2}=.12$. Neither the effect of electrodes nor the tone $\times$ electrodes interaction were significant $\left(F_{\mathrm{S}}<1.0\right)$. As for the EDAN, no effects of tone were found when the tone was presented with TCOA $500 \mathrm{~ms}, F \leq 1.41, p \geq .24$.

\section{Discussion}

\section{Behavioral results}

The obtained behavioral results showed the usual effect of attentional orienting and the expected alerting $\times$ orienting interaction. Responses were generally faster when the central arrows cued the spatial location of the following target compared with responses in the trials with the neutral cue. This indicates more efficient target processing at attended location (Carrasco, 2011; Posner, 2014). Importantly, this orienting effect was increased when the tone was presented $100 \mathrm{~ms}$ before the cue, indicating that phasic alerting facilitated the process of orienting, in line with the previous behavioral findings (Callejas et al., 2005; Callejas et al., 2004; Fuentes \& Campoy, 2008; Ishigami et al., 2016).

\section{Event-related lateralizations}

To investigate the neural basis of the anticipatory orienting of attention toward target location within the cue-target interval, we calculated event-related lateralizations (ERLs) of the EEG (van der Lubbe et al., 2006; Wascher \& Wauschkuhn, 1996). When the cue was neutral, no significant ERLs were observed, indicating no spatial shifts of attention before target onset, as expected. When the valid cue was presented, a characteristic sequence of ERLs was found, composed of three early cue processing-related components, and subsequent two preparatory orienting-related components, EDAN and LDAP.

The first three components occurred within the first $300 \mathrm{~ms}$ of the cue-target interval: a negative peak at about $100 \mathrm{~ms}$ after cue onset, a positive peak at $160 \mathrm{~ms}$, and a second negative peak at about $220 \mathrm{~ms}$. Such early components are usually not present in typical directing attention-related ERLs (Praamstra \& Kourtis, 2010; van der Lubbe et al., 2019; van der Lubbe \& Utzerath, 2013; Wascher \& Wauschkuhn, 1996), but similar early posterior ERL peaks have been observed in some spatial cueing studies (e.g., Banerjee, Frey, Molholm, \& Foxe, 2015; Jongen, Smulders, \& der Heiden, 2007) and in experiments with different cueing or priming procedures (e.g., Schneider, Hoffmann, \& Wascher, 2014; Verleger, vel Grajewska, \& Jaśkowski, 2012). The time course of these waveforms, as well as their occipitotemporal distribution centered at PO7-8, fit the description of selection-related early posterior contralateral components $(\mathrm{N} 1 \mathrm{pc}, \mathrm{P} 1 \mathrm{pc}$, and $\mathrm{N} 2 \mathrm{pc}$; see Verleger et al., 2012, for a review). Thus, accordingly, we tentatively classified them as cue processing-related $\mathrm{N} 1 \mathrm{pc}, \mathrm{P} 1 \mathrm{pc}$, and N2pc. While certainly related to a perceptual asymmetry of the arrow cues used in the present study (such early ERLs are usually not observed when fully symmetrical symbolic cues are presented; e.g., van der Lubbe et al., 2019; van Velzen \& Eimer, 2003), these components seem to reflect 
initial attentional operations of stimulus-driven detection and selection of task-relevant cue features (cf. van Velzen \& Eimer, 2003; Verleger et al., 2012).

The early ERLs were followed by another negative peak at about $320 \mathrm{~ms}$ after cue onset. We classified this peak as EDAN, because it occurred within the EDAN time frame (Harter et al., 1989; Hopf \& Mangun, 2000; van der Lubbe et al., 2006) and had the EDAN distribution, which distinguish it from the preceding cue-related N2pc. Praamstra and Kourtis (2010) have demonstrated that while the cue selection-related N2pc has an occipitotemporal distribution, the proper, preparatory orienting-related EDAN has a wider, occipitoparietal distribution. In line with this evidence, the first of these peaks seems to be indeed a cue-related N2pc representing selection of the task-relevant features of arrows indicating to-be-attended side (van Velzen \& Eimer, 2003), whereas the second one is the EDAN, plausibly representing initiation of voluntary orienting toward the cued location (Hopf \& Mangun, 2000; Praamstra \& Kourtis, 2010).

The last of the observed ERLs was the LDAP, a positive deflection emerged about $350 \mathrm{~ms}$ after cue onset and lasted until the onset of target-evoked activity, which corresponds with the LDAP findings of other studies (Harter et al., 1989; Hopf \& Mangun, 2000; Praamstra \& Kourtis, 2010). The LDAP had a typical occipitotemporal scalp distribution, most likely reflecting an enhanced neuronal activity in the ventral extrastriate visual cortex representing the attended region of visual space (Hopf \& Mangun, 2000; van der Lubbe \& Utzerath, 2013). This preparatory modulation has been shown to be produced by top-down projections from the frontoparietal orienting network (Baldauf \& Desimone, 2014; Buffalo, Fries, Landman, Liang, \& Desimone, 2010; Corbetta et al., 2005; Vossel, Weidner, Driver, Friston, \& Fink, 2012). Finally, no anterior ERL components (i.e., an anterior directing attention negativity or ADAN) were found in the present study (see Hopf \& Mangun, 2000; van der Lubbe et al., 2019; van der Lubbe \& Utzerath, 2013, for similar results).

\section{Effects of phasic alerting on orienting}

Crucially, the EDAN and LDAP components were significantly modulated by the tone presented $100 \mathrm{~ms}$ before the orienting cue. The EDAN had a smaller amplitude and a marginally shorter latency, whereas the LDAP had a larger amplitude and a shorter onset latency under the phasic alerting condition.

The LDAP results seem straightforward - they suggest that alerting both accelerated and enhanced the orienting-related preparatory modulations within the visual cortex. Thereby, the results prompt a compromise between the accelerating hypothesis and the enhancing hypothesis. Indeed, the two accounts do not have to be mutually exclusive. The LDAP results suggest that alerting may facilitate orienting of attention in terms of both speed and strength of the modulatory topdown projections from the attention network. This would also indicate that the previous seemingly contradictory behavioral findings, reported by Callejas et al. (2005; Callejas et al., 2004) on the one hand, and by Fuentes and Campoy (2008) on the other (see the Introduction), were all partially correct. When taken together, these behavioral results are in agreement with the present findings.

Less clear is the observed effect of alerting on the EDAN and its relationship to the LDAP. The smaller EDAN amplitude under the alerting condition may be due to an attenuation of EDAN by the faster emerging LDAP (see Jongen et al., 2007; Praamstra \& Kourtis, 2010, for suggestions of such attenuation or overlap between the two ERLs). The opposite might also be true - the reduced amplitude of the EDAN might have resulted in the shorter latency of the LDAPalthough this does not explain why the EDAN was smaller under the alerting condition. Most plausibly, however, the sequence of alerting effects on the EDAN and LDAP reflect modulation of two subsequent attentional operations. As mentioned in the introduction, EDAN is thought to reflect an initiation of cue-directed orienting processes within the frontoparietal attention network (Hopf \& Mangun, 2000; Praamstra \& Kourtis, 2010) — that is, "the control of selection" (Luck \& Gold, 2008), whereas LDAP reflects an "implementation of selection" within the visual system. Referring to the "spotlight in the brain" metaphor of attention (cf. FernandezDuque \& Johnson, 1999), Luck and Gold (2008) have described the control of selection as "analogous to pointing the beam in the correct direction," whereas the implementation of selection as "analogous to the strength of the beam" (p. 37). Assuming that alerting facilitates the whole orienting process, the pointing of the beam in the correct direction might be less effortful under the alerting condition, resulting in a smaller amplitude and a shorter latency of EDAN. At the same time, the beam illuminating the selected stimulus within the visual cortex would be stronger, thus more effective, resulting in a larger amplitude and a shorter latency of LDAP. Of course, such an interpretation of the relationship between ERL amplitudes and processes' efficiency is speculative, and further experimental evidence is needed here. Especially, the smaller EDAN amplitude under the alerting condition needs to be explained.

Another question that remains to be answered is whether alerting affects orienting through direct functional connections between the neural networks controlling these attentional operations (attention networks; Petersen \& Posner, 2012; Posner \& Rothbart, 2007), as it is often assumed (e.g., Bukowski, Asanowicz, Marzecová, \& Lupiáñez, 2015; Callejas et al., 2005; Callejas et al., 2004; Fan et al., 2009; Posner, 2008; Spagna et al., 2014), or whether the interaction occurs in an indirect way - that is, via other brain systems or mechanisms 
(cf. Böckler, Alpay, \& Stürmer, 2011; Nieuwenhuis \& de Kleijn, 2013; Weinbach \& Henik, 2012b). For instance, alerting may accelerate the orienting operation by direct bottom-up projections from the alerting network, presumably via norepinephrine projections from the locus coeruleus (Aston-Jones \& Cohen, 2005; Petersen \& Posner, 2012) to the frontoparietal orienting network. Alternatively, alerting may increase the speed and sensitivity of perceptual processing (Petersen, Petersen, Bundesen, Vangkilde, \& Habekost, 2017), thereby indirectly facilitating and enhancing the magnitude of the orienting-related modulations within the visual system.

\section{Conclusion}

Two accounts of the effect of phasic alerting on spatial orienting have been put forward: the accelerating hypothesis and the enhancing hypothesis. To test these hypotheses, we used a variant of endogenous spatial cueing task with a phasic alerting manipulation, and measured preparatory orientingrelated event-related lateralizations (ERLs) of the EEG. While behavioral results confirmed the presence of the alerting-orienting interaction, the ERL results showed that this interaction may be due to alerting-induced modulations of an occipitotemporal ERL component called a late directing attention positivity (LDAP). Importantly, both the latency and the amplitude of the LDAP were affected. These findings suggest that phasic alerting both accelerates and enhances orienting-related preparatory modulations within the ventral visual cortex, thereby favoring a compromise between the two hypotheses.

Acknowledgements The present work was supported by Grant 2016/22/ E/HS6/00139 awarded from the National Science Centre of Poland to D.A. The authors thank Magdalena Gorgoń and Mikołaj Compa for their contribution in data collection.

Open practices statement The data that support the findings of this study are available from the corresponding author, upon reasonable request. The experiment was not preregistered.

\section{References}

Asanowicz, D., \& Marzecová, A. (2017). Differential effects of phasic and tonic alerting on the efficiency of executive attention. Acta Psychologica, 176, 58-70. doi:https://doi.org/10.1016/j.actpsy. 2017.03.004

Aston-Jones, G., \& Cohen, J. D. (2005). An integrative theory of locus coeruleus-norepinephrine function: Adaptive gain and optimal performance. Annual Review of Neuroscience, 28(1), 403-450. doi: https://doi.org/10.1146/annurev.neuro.28.061604.135709

Baldauf, D., \& Desimone, R. (2014). Neural mechanisms of object-based attention. Science, 344(6182), 424-427. doi:https://doi.org/10.1126/ science. 1247003
Banerjee, S., Frey, H., Molholm, S., \& Foxe, J. J. (2015). Interests shape how adolescents pay attention: The interaction of motivation and top-down attentional processes in biasing sensory activations to anticipated events. European Journal of Neuroscience, 41(6), 818834. doi:https://doi.org/10.1111/ejn.12810

Böckler, A., Alpay, G., \& Stürmer, B. (2011). Accessory stimuli affect the emergence of conflict, not conflict control. Experimental Psychology, 58(2), 102-109. doi:https://doi.org/10.1027/16183169/a000073

Buffalo, E. A., Fries, P., Landman, R., Liang, H., \& Desimone, R. (2010). A backward progression of attentional effects in the ventral stream. Proceedings of the National Academy of Sciences of the United States of America, 107(1), 361-365. doi:https://doi.org/10.1073/ pnas.0907658106

Bukowski, M., Asanowicz, D., Marzecová, A., \& Lupiáñez, J. (2015). Limits of control: The effects of uncontrollability experiences on the efficiency of attentional control. Acta Psychologica, 154, 43-53. doi:https://doi.org/10.1016/j.actpsy.2014.11.005

Callejas, A., Lupiàñez, J., Funes, M., \& Tudela, P. (2005). Modulations among the alerting, orienting and executive control networks. Experimental Brain Research, 167(1), 27-37. doi:https://doi.org/ 10.1007/s00221-005-2365-z

Callejas, A., Lupiáñez, J., \& Tudela, P. (2004). The three attentional networks: On their independence and interactions. Brain and Cognition, 54(3), 225-227. doi:https://doi.org/10.1016/j.bandc. 2004.02.012

Carrasco, M. (2011). Visual attention: The past 25 years. Vision Research, 51(13), 1484-1525. doi:https://doi.org/10.1016/j.visres.2011.04. 012

Chica, A. B., de Schotten, M., Toba, M., Malhotra, P., Lupiáñez, J., \& Bartolomeo, P. (2012). Attention networks and their interactions after right-hemisphere damage. Cortex, 48(6), 654-663. doi: https://doi.org/10.1016/j.cortex.2011.01.009

Corbetta, M., \& Shulman, G. L. (2002). Control of goal-directed and stimulus-driven attention in the brain. Nature Reviews Neuroscience, 3(3), 201-215. doi:https://doi.org/10.1038/nrn755

Corbetta, M., Tansy, A. P., Stanley, C. M., Astafiev, S. V., Snyder, A. Z., \& Shulman, G. L. (2005). A functional MRI study of preparatory signals for spatial location and objects. Neuropsychologia, 43(14), 2041-2056. doi:https://doi.org/10.1016/j.neuropsychologia.2005. 03.020

Fan, J., Gu, X., Guise, K. G., Liu, X., Fossella, J., Wang, H., \& Posner, M. I. (2009). Testing the behavioral interaction and integration of attentional networks. Brain and Cognition, 70(2), 209-220. doi:https:// doi.org/10.1016/j.bandc.2009.02.002

Fernandez-Duque, D., \& Johnson, M. (1999). Attention metaphors: How metaphors guide the cognitive psychology of attention. Cognitive Science, 23(1), 83-116. doi:https://doi.org/10.1207/ s15516709 $\operatorname{cog} 2301 \_$

Fernandez-Duque, D., \& Posner, M. I. (1997). Relating the mechanisms of orienting and alerting. Neuropsychologia, 35(4), 477-486. doi: https://doi.org/10.1016/S0028-3932(96)00103-0

Fuentes, L. J., \& Campoy, G. (2008). The time course of alerting effect over orienting in the attention network test. Experimental Brain Research, 185(4), 667-672. doi:https://doi.org/10.1007/s00221007-1193-8

Gratton, G., Coles, M. G., Sirevaag, E. J., Eriksen, C. W., \& Donchin, E. (1988). Pre- and poststimulus activation of response channels: A psychophysiological analysis. Journal of Experimental Psychology: Human Perception and Performance, 14(3), 331334. doi:https://doi.org/10.1037//0096-1523.14.3.331

Hackley, S. A., \& Valle-Inclán, F. (2003). Which stages of processing are speeded by a warning signal? Biological Psychology, 64(1/2), 2745. doi:https://doi.org/10.1016/S0301-0511(03)00101-7

Harter, R. M., Miller, S. L., Price, N. J., LaLonde, M. E., \& Keyes, A. L. (1989). Neural processes involved in directing attention. Journal of 
Cognitive Neuroscience, 1(3), 223-237. doi:https://doi.org/10.1162/ jocn.1989.1.3.223

Hopf, J.-M., \& Mangun, G. (2000). Shifting visual attention in space: An electrophysiological analysis using high spatial resolution mapping. Clinical Neurophysiology, 111(7), 1241-1257. doi:https://doi.org/ 10.1016/s1388-2457(00)00313-8

Ishigami, Y., Eskes, G. A., Tyndall, A. V., Longman, S. R., Drogos, L. L., \& Poulin, M. J. (2016). The Attention Network Test-Interaction (ANT-I): Reliability and validity in healthy older adults. Experimental Brain Research, 234(3), 815-827. doi:https://doi. org/10.1007/s00221-015-4493-4

Jongen, E. M., Smulders, F. T., \& der Heiden, J. S. (2007). Lateralized ERP components related to spatial orienting: Discriminating the direction of attention from processing sensory aspects of the cue. Psychophysiology, 44(6), 968-986. doi:https://doi.org/10.1111/j. 1469-8986.2007.00557.x

Jung, T., Makeig, S., Humphries, C., Lee, T., McKeown, M. J., Iragui, V., \& Sejnowski, T. J. (2000). Removing electroencephalographic artifacts by blind source separation. Psychophysiology, 37(2), 163-178.

Kiesel, A., Miller, J., Jolicoeur, P., \& Brisson, B. (2008). Measurement of ERP latency differences: A comparison of single-participant and jackknife-based scoring methods. Psychophysiology, 45(2), 250 74. doi:https://doi.org/10.1111/j.1469-8986.2007.00618.x

Lasaponara, S., Chica, A. B., Lecce, F., Lupianez, J., \& Doricchi, F. (2011). ERP evidence for selective drop in attentional costs in uncertain environments: Challenging a purely premotor account of covert orienting of attention. Neuropsychologia, 49(9), 26482657. doi:https://doi.org/10.1016/j.neuropsychologia.2011.05.012

Luck, S. J. (2014). An introduction to the event-related potential technique (2nd ed.). Cambridge, MA: MIT Press.

Nieuwenhuis, S., \& de Kleijn, R. (2013). The impact of alertness on cognitive control. Journal of Experimental Psychology: Human Perception and Performance, 39(6), 1797. doi:https://doi.org/10. 1037/a0033980

Nobre, A. C. K., Rohenkohl, G., \& Stokes, M. G. (2012). Nervous anticipation: Top-down biasing across space and time. In M. I. Posner (Ed.), Cognitive neuroscience of attention (2nd ed., pp. 159-186). New York, NY: Guilford Press.

Petersen, A., Petersen, A. H., Bundesen, C., Vangkilde, S., \& Habekost, T. (2017). The effect of phasic auditory alerting on visual perception. Cognition, 165, 73-81. doi:https://doi.org/10.1016/j.cognition. 2017.04.004

Petersen, S. E., \& Posner, M. I. (2012). The attention system of the human brain: 20 years after. Annual Review of Neuroscience, 35(1), 73-89. doi:https://doi.org/10.1146/annurev-neuro-062111-150525

Posner, M. I. (1978). Mental chronometry: Chronometric explorations of mind. Hillsdale, NJ: Erlbaum.

Posner, M. I. (2008). Measuring alertness. Annals of the New York Academy of Sciences, 1129(1), 193-199. doi:https://doi.org/10. 1196/annals.1417.011

Posner, M. I. (2012). Progress in attention research 2004-2011. In M. I. Posner (Ed.), Cognitive neuroscience of attention (2nd ed., pp. 110). New York, NY: Guilford Press.

Posner, M. I. (2014). Orienting of attention: Then and now. Quarterly Journal of Experimental Psychology, 69(10), 1864-1875. doi: https://doi.org/10.1080/17470218.2014.937446

Posner, M. I., \& Fan, J. (2008). Attention as an organ system. In J. R. Pomerantz (Ed.), Topics in integrative neuroscience: From cells to cognition (pp. 31-61). New York, NY: Cambridge University Press.

Posner, M. I., \& Rothbart, M. K. (2007). Research on attention networks as a model for the integration of psychological science. Annual Review of Psychology, 58(1), 1-23. doi:https://doi.org/10.1146/ annurev.psych.58.110405.085516
Praamstra, P., \& Kourtis, D. (2010). An early parietal ERP component of the frontoparietal system: EDAN $\neq N 2 p c$. Brain Research, 1317, 203-210. doi:https://doi.org/10.1016/j.brainres.2009.12.090

Schneider, D., Hoffmann, S., \& Wascher, E. (2014). Sustained posterior contralateral activity indicates re-entrant target processing in visual change detection: An EEG study. Frontiers in Human Neuroscience, 8(247), 1-10. doi:https://doi.org/10.3389/fnhum.2014.00247

Spagna, A., Martella, D., Sebastiani, M., Maccari, L., Marotta, A., \& Casagrande, M. (2014). Efficiency and interactions of alerting, orienting and executive networks: The impact of imperative stimulus type. Acta Psychologica, 148C, 209-215. doi:https://doi.org/10. 1016/j.actpsy.2014.02.007

Talsma, D., Wijers, A. A., Klaver, P., \& Mulder, G. (2001). Working memory processes show different degrees of lateralization: Evidence from event-related potentials. Psychophysiology, 38(3), 425-439. doi:https://doi.org/10.1111/1469-8986.3830425

van der Lubbe, R. H., de Kleine, E., \& Rataj, K. K. (2019). Dyslexic individuals orient but do not sustain visual attention: Electrophysiological support from the lower and upper alpha bands. Neuropsychologia, 125, 30-41. doi:https://doi.org/10.1016/j. neuropsychologia.2019.01.013

van der Lubbe, R. H., Neggers, S., Verleger, R., \& Kenemans, L. J. (2006). Spatiotemporal overlap between brain activation related to saccade preparation and attentional orienting. Brain Research, 1072, 133-152. doi:https://doi.org/10.1016/j.brainres.2005.11.087

van der Lubbe, R. H., \& Utzerath, C. (2013). Lateralized power spectra of the EEG as an index of visuospatial attention. Advances in Cognitive Psychology, 9(4), 184-201. doi:https://doi.org/10.2478/v10053008-0144-7

van Velzen, J., \& Eimer, M. (2003). Early posterior ERP components do not reflect the control of attentional shifts toward expected peripheral events. Psychophysiology, 40(5), 827-831. doi:https://doi.org/ 10.1111/1469-8986.00083

Verleger, R., Vel Grajewska, B., \& Jaśkowski, P. (2012). Time-course of hemispheric preference for processing contralateral relevant shapes: $\mathrm{P} 1 \mathrm{pc}, \mathrm{N} 1 \mathrm{pc}, \mathrm{N} 2 \mathrm{pc}, \mathrm{N} 3 \mathrm{pc}$. Advances in Cognitive Psychology, 8(1), 19-28. doi:https://doi.org/10.2478/v10053-008-0098-9

Vossel, S., Weidner, R., Driver, J., Friston, K. J., \& Fink, G. R. (2012). Deconstructing the architecture of dorsal and ventral attention systems with dynamic causal modeling. The Journal of Neuroscience, 32(31), 10637-10648. doi:https://doi.org/10.1523/JNEUROSCI. 0414-12.2012

Wascher, E., \& Wauschkuhn, B. (1996). The interaction of stimulus- and response-related processes measured by event-related lateralizations of the EEG. Electroencephalography and Clinical Neurophysiology, 99(2), 149-162. doi:https://doi.org/10.1016/0013-4694(96)95602-3

Weinbach, N., \& Henik, A. (2012a). Temporal orienting and alertingThe same or different? Frontiers in Psychology, 3, 236. doi:https:// doi.org/10.3389/fpsyg.2012.00236

Weinbach, N., \& Henik, A. (2012b). The relationship between alertness and executive control. Journal of Experimental Psychology: Human Perception and Performance, 38(6), 1530. doi:https://doi.org/10. 1037/a0027875

Weinbach, N., \& Henik, A. (2013). The interaction between alerting and executive control: Dissociating phasic arousal and temporal expectancy. Attention, Perception, \& Psychophysics, 75(7), 1374-1381. doi:https://doi.org/10.3758/s13414-013-0501-6

Publisher's note Springer Nature remains neutral with regard to jurisdictional claims in published maps and institutional affiliations. 